\title{
Variation in the provision of chemotherapy for colorectal cancer
}

\author{
Alice McLeod
}

\begin{abstract}
Objective-To quantify the impact of patient, area and hospital characteristics on variations in the provision of chemotherapy for colorectal cancer.

Subjects-Incident cases of colorectal cancer (ICD 153-154), aged under 75 years and resident in Scotland, derived from linked hospital discharge records and death records for the period January 1990 to June 1994. The final analysis was carried out on 7852 patients resident in 823 areas and first admitted to one of 59 hospitals.
\end{abstract}

Main outcome measure-Whether a patient received chemotherapy (OPCS4 procedure code $\mathrm{X} 35.2$ ) during any hospital episode in the six months after their first admission.

Methods-Multilevel logistic regression to separate effects of patients, areas and hospitals.

Results-During the study period, $8 \%$ $(n=626)$ of the study population received chemotherapy within six months of their first admission. Adjusting for comorbidities and emergency admissions, both age and deprivation were significantly associated with the treatment. The odds ratios $(\mathrm{OR})$ of chemotherapy relative to patients aged 65-74 were 2.13 and 4.50 for patients aged 55-64 and under 55 respectively. Relative to patients resident in the most affluent areas, the OR of chemotherapy for patients resident in the most deprived areas was 0.73 . Area level availability of the treatment was not significantly associated with a patient's odds of receiving the treatment while on site provision of chemotherapy at the hospital of first admission was $(O R=4.32)$. There was significant unexplained variation between hospitals of first admission but not between areas of residence; between hospital variation decreased by $22 \%$ during the study period.

Conclusion-Differences according to age may reflect both clinical and patient decisions regarding the benefits of the treatment relative to its toxicity. Lower treatment rates in deprived areas may indicate inequitable access to services. Hospital differences may reflect consultant effects and it would be expected that these should decrease now that the efficacy of the treatment has been recognised and guidelines have been issued. (F Epidemiol Community Health 1999;53:775-781)
In 1990, the King's Fund consensus statement on colorectal cancer included the recommendation that patients should be enrolled in trials to establish the efficacy of adjuvant chemotherapy. ${ }^{1}$ At that time, there was some evidence that chemotherapy for advanced (Dukes's stage C) colorectal cancer may improve survival and reduce the risk of recurrence, ${ }^{2}$ or at least help in the palliation of the most advanced cases (stage D). ${ }^{1}$ More recent guidelines and reviews have restated the benefits of chemotherapy for advanced stages of the disease, although results from ongoing trials are still required to establish optimal treatment regimens and the effectiveness of the treatment for less advanced cases (stage B). ${ }^{34}$ The distribution of stage at presentation is not currently known in Scotland; however, there is evidence to suggest that around $40-60 \%$ of new cases will present with advanced colorectal cancer. ${ }^{4-6}$

Colorectal cancer is currently the second and third most common cancer in Scottish men and women respectively, resulting in around 3000 new cases annually. ${ }^{7}$ Even before the introduction of the latest clinical guidelines in Scotland, important shifts in colorectal cancer care were emerging; acute inpatient and daycase episodes for colorectal cancer increased from 6688 in 1990 to 11434 in 1994 while incidence stayed relatively constant. Around $70 \%$ of this increase was attributable to episodes in which the principal procedure was chemotherapy. The changes in Scotland are similar to those recently reported for a patient population in southern England and show that increased hospital activity is a result of patients receiving more complex packages of care such as chemotherapy after surgery. ${ }^{8}$ While these shifts in treatment practice have important implications for the planning and provision of future cancer services, there is also an important issue of current variations in the provision of a new technology. One of the key findings from the study of the English population was that the substantial increases in activity were limited to a small proportion of patients.

Equitable provision of services and access to them has received limited attention in British health services research. It has been argued that inequitable health care contributes less to health inequalities in Britain than factors such as housing, employment and education. ${ }^{9}$ However, Benzeval et al point out that while, in principle, the NHS provides equal access for equal need it is important to evaluate the reality of this assertion. ${ }^{9}$ There is conflicting evidence to support equal access among different social 
groups, especially in terms of acute services. ${ }^{9}{ }^{10}$ This is perhaps not surprising given that the pathways linking need and provision are complex and the methods used to measure and quantify their associations vary from study to study. It is also likely that the size and significance of any inequalities in access will vary over time and across areas, as well as according to the particular service under study. Indeed, rather than specific studies of inequitable access, it has been argued that what is required is a means of routinely monitoring the variety of services provided within the NHS to identify systematic variation in the association between need and provision..$^{911}$

Ben-Shlomo and Chaturvedi illustrate such a method using routine hospital episode data on coronary artery bypass grafts and show how the provision of the procedure varies according to need, proxied by ischaemic heart disease mortality, and deprivation at the small area level. ${ }^{11} \mathrm{~A}$ more in depth study of variation in access to health care is presented by Gatsonis et $a l$ in their analysis of coronary angiography for acute myocardial infarction in a national American cohort. ${ }^{12}$ These authors explore the complexity of inequitable access by modelling not only the impact on utilisation of individual characteristics but also those operating at the area level, such as the availability of the procedure in the state of residence. Furthermore, the method used allows them to show that the impact of individual characteristics varies according to context with, for example, the effect of race being more pronounced in southern states. The difference between the two approaches is that the former uses individual attributes aggregated to the level of area to explore variation in access ${ }^{11}$ while the latter explicitly models the effects of both people and areas and how access varies between them. ${ }^{12}$ This paper differs from both in that, while considering patient attributes, it also takes account of both area and hospital of first admission and illustrates how the method may be used to monitor variations over time. These methods are applied to the example of chemotherapy for colorectal cancer patients in Scotland in the period 1990-94.

\section{Methods}

DATA

Analyses were carried out using routinely available hospital discharge data. In Scotland, all episodes of hospital care are reported on a standard discharge form (Scottish Morbidity Record 1- SMR1) and assembled centrally by the Information and Statistics Division of the Common Services Agency (ISD). ISD has linked these data using probability matching to provide a person-based record of inpatient care that is also linked to General Register Office death records. ${ }^{13}$ A total of 15016 people were identified from the linked SMR1 dataset as having had their first acute inpatient treatment for colorectal cancer (ICD-9 153-154) in the period 1990-94. Patients aged over 75 $(n=5860)$ were excluded from the analysis on the basis of empirical evidence suggesting chemotherapy was unlikely to be provided for older patients ( $n=57$ received chemotherapy). It was necessary to define a treatment period to estimate how the provision of chemotherapy was changing over time. Just over $70 \%$ of patients who received chemotherapy did so within six months of their first admission and this was chosen as a suitable treatment period. This meant patients whose first hospital episode for colorectal cancer was after June 1994 ( $n=997$ ) did not have adequate follow up in the available data and they were therefore excluded from the analysis. Finally, patients identified as being resident outside Scotland $(n=45)$ were excluded on the basis that their complete treatment for the disease may not have been carried out solely in Scotland. This resulted in a total of 8114 patients eligible for inclusion in the analysis. The main outcome measure was whether or not the patient received chemotherapy in either their first hospital episode or in any colorectal cancer episode in the six months after their first admission. Patients were classed as having received chemotherapy if the OPCS4 procedure code X35.2 (intravenous chemotherapy) was recorded as a principal or subsidiary treatment in any completed episode within the treatment period. ${ }^{14}$

The patient's age, sex and marital status, recorded on the SMR1 form, were each used to investigate variations in chemotherapy provision. Dukes's staging of the cancer is one of the most important determinants of the need for chemotherapy; however, this information is not available routinely. Instead, two variables were used to summarise clinical factors relating to the disease: type of admission when first treated (emergency or elective) and secondary diagnosis at first admission. Secondary diagnoses were grouped into ICD9 chapters for the purpose of analysis and chapters forming less than $0.5 \%$ of the sample were grouped into a miscellaneous category. The majority of patients had no recorded secondary diagnosis $(66.3 \%)$ while the largest comorbidity group was other neoplasms $(17.5 \%)$. In order of prevalence the remaining comorbidity groups were diseases of the digestive system $(6.1 \%)$, diseases of the circulatory system $(2.2 \%)$, injury and poisoning $(1.4 \%)$, diseases of the blood $(1.3 \%)$, symptoms and signs (1.2\%), miscellaneous comorbidities $(1.1 \%)$, diseases of the respiratory system $(0.9 \%)$ and endocrine disorders $(0.8 \%)$. A binary indicator of whether the patient died within the six month treatment period was also included in the model.

The remaining 8114 patients were first admitted to 94 Scottish hospitals between January 1990 and June 1994 and the majority $(85 \%)$ completed their six month treatment at the hospital of first admission. One hospital variable was constructed from the data to measure on site availability of chemotherapy; hospitals providing more than five episodes of care in which intravenous chemotherapy was recorded were classed as providing on site treatment. ${ }^{12}$ This measure of availability was weighted by the number of years in which chemotherapy had been recorded divided by 
the number of years the hospital had been operating during the five year study period. Hospitals that provided the treatment throughout the study period had an availability measure of one, while those that had been operating throughout the study period but only recorded chemotherapy for one year had an availability measure of 0.2 . Hospitals admitting very few colorectal cancer patients $(<5)$ were excluded on the grounds that, by definition, they would be unlikely to be classed as providing on site chemotherapy as well as the fact that any inferences regarding their effect on a patient's treatment would be limited. This resulted in a total of 59 hospitals with a loss of only 55 patients.

Included on the SMR1 record is the patient's postcode sector of residence and this was used to group patients into areas. The majority of patients were recorded as being resident in one of 854 postcode sectors while 82 patients had an unknown area of residence. Three area characteristics were used to explore variation in chemotherapy provision; deprivation, rurality and an empirical measure of availability. Deprivation was measured using Carstairs' 1991 scores grouped into quartiles ${ }^{15}$; higher scores indicate greater deprivation, therefore, the upper quartile contained the most deprived areas and the lower quartile, the most affluent. Rurality was measured by population density (persons per hectare) based on the 1991 census. This measure was dichotomised on the basis of its bimodal distribution and areas with less than six persons per hectare were classed as rural. Area level availability was defined as the proportion of patients in the area who were first admitted to a hospital that provided on site chemotherapy. ${ }^{12}$ Information on deprivation and rurality was missing for the 82 patients of unknown area of residence and for 125 patients resident in 31 postcode sectors that were created after the 1991 census. Normally, it would be possible to include these patients as a separate category for whom deprivation/ population density was missing; however, because none of the 207 patients received chemotherapy it was necessary to exclude them from the analysis. The final sample structure on which the following results are based is 7852 patients, resident in 823 areas and first admitted to one of 59 hospitals.

STATISTICAL ANALYSIS

The aims of this study were to estimate the impact of patient, area and hospital characteristics on the probability of receiving chemotherapy, to identify areas and hospitals for which the outcome was significantly increased or decreased from that which would be expected, and to estimate the uptake of chemotherapy over time. The data formed a hierarchical structure of patients nested within areas and hospitals of first admission and explanatory variables were measured at each of these three levels. Although patients could receive their treatment at more than one hospital, the hospital of first admission was used to define the hierarchy rather than hospital of treatment because the former would provide a
KEY POINTS

- Older patients and those living in more deprived areas were less likely to receive chemotherapy.

- On site provision of chemotherapy at the hospital of first admission significantly increased a patient's odds of receiving the treatment.

- Variation in the provision of chemotherapy was greater between hospitals than between areas of residence.

- Unexplained hospital variation decreased by $22 \%$ between 1990 and 1994 .

better indicator of the consultant to which a patient was first referred. The utility of multilevel, or hierarchical, models for the analysis of such complex data structures is well documented and the methods have been applied in a variety of health settings. ${ }^{16}$ The methods are based on the principle that people are naturally grouped into units, for example, geographical or organisational, and that characteristics of these units may influence the outcomes of their members. Within the context of this research, Gatsonis et al provide a comprehensive discussion of the methodology and the benefits it proffers over traditional single level analyses. ${ }^{12}$ The data were analysed within a multilevel framework and all analyses were carried out within the statistical software package MLwiN-this approach enabled the analysis of both areas and hospitals that did not form a strict hierarchy because people from the same area may attend different hospitals. ${ }^{17}$ Multilevel logistic regression was used to model the binary outcome and models were estimated using restricted iterative generalised least squares. The effects of patient, area and hospital characteristics were estimated as the increase in odds of chemotherapy relative to an appropriate baseline group, and the additional, unexplained effects of areas and hospitals were estimated as the increase in odds relative to the Scottish average.

\section{Results}

CHARACTERISTICS OF THE STUDY POPULATION A total of 626 patients (8\%) from the 7852 received chemotherapy in the first six months of their treatment. There was a median of nine colorectal cancer cases per area (range 1-32) and a median of 105 first admissions per hospital (range 5-624). The percentage of patients receiving chemotherapy ranged from $0 \%$ to $100 \%$ across areas with a median of $0 \%$, and from $0 \%$ to $68 \%$ across hospitals with a median of $3 \%$. The median number of areas from which hospitals received first admissions was 20 (range 2-131) while the median number of hospitals to which patients resident in the same area were first admitted was 2 (range 1-6). Only 19 of the 59 hospitals provided on site chemotherapy; however, $56 \%$ of patients were first admitted to one of these hospitals and they were attended at least once by $61 \%$ of patients at some point in their six months of treatment. 
Table 1 Association between patient, area and hospital characteristics and chemotherapy

\begin{tabular}{|c|c|c|c|c|c|}
\hline Patient characteristics (baseline) & & $\begin{array}{l}\text { Unadjusted } \\
\text { OR } \\
\text { chemotherapy }\end{array}$ & $95 \% C I$ & $\begin{array}{l}\text { Adjusted } \\
\text { OR } \\
\text { chemotherapy }\end{array}$ & $95 \% C I$ \\
\hline \multirow{2}{*}{ Age (65-74) } & $0-54$ & 4.98 & $(3.99,6.21)$ & 4.50 & $(3.58,5.66)$ \\
\hline & $55-64$ & 2.31 & $(1.86,2.86)$ & 2.13 & $(1.71,2.65)$ \\
\hline Sex (males) & females & 1.05 & $(0.89,1.25)$ & 1.13 & $(0.94,1.35)$ \\
\hline \multirow{3}{*}{ Marital status (single) } & married & 1.33 & $(0.96,1.84)$ & 1.35 & $(0.96,1.89)$ \\
\hline & widowed & 0.54 & $(0.35,0.86)$ & 0.78 & $(0.48,1.24)$ \\
\hline & $\begin{array}{l}\text { other (including } \\
\text { divorced) }\end{array}$ & 1.74 & $(1.20,2.53)$ & 1.76 & $(1.20,2.60)$ \\
\hline Admission (elective) & emergency & 0.80 & $(0.66,0.98)$ & 0.78 & $(0.63,0.97)$ \\
\hline \multirow[t]{10}{*}{ Secondary diagnosis (none) } & neoplasms & 1.97 & $(1.61,2.41)$ & 1.99 & $(1.60,2.48)$ \\
\hline & endocrine disorders & 0.91 & $(0.32,2.61)$ & 1.04 & $(0.36,3.04)$ \\
\hline & diseases of blood & 0.22 & $(0.05,0.93)$ & 0.25 & $(0.06,1.09)$ \\
\hline & circulatory disease & 0.16 & $(0.04,0.66)$ & 0.19 & $(0.05,0.82)$ \\
\hline & respiratory disease & 0.37 & $(0.09,1.61)$ & 0.49 & $(0.11,2.11)$ \\
\hline & digestive system disease & 0.58 & $(0.37,0.91)$ & 0.63 & $(0.40,1.00)$ \\
\hline & genitourinary disease & 0.27 & $(0.06,1.14)$ & 0.32 & $(0.07,1.37)$ \\
\hline & symptoms and signs & 0.83 & $(0.34,1.99)$ & 0.88 & $(0.36,2.16)$ \\
\hline & injury and poisoning & 0.22 & $(0.05,0.93)$ & 0.23 & $(0.05,0.99)$ \\
\hline & other & 0.13 & $(0.02,1.00)$ & 0.13 & $(0.02,1.00)$ \\
\hline $\begin{array}{l}\text { Died within treatment period (no) } \\
\text { Area characteristics }\end{array}$ & yes & 1.08 & $(0.88,1.34)$ & 1.06 & $(0.84,1.34)$ \\
\hline \multirow[t]{3}{*}{ Deprivation (most affluent quartile) } & mid-affluent quartile & 1.01 & $(0.78,1.32)$ & 1.16 & $(0.88,1.52)$ \\
\hline & mid-deprived quartile & 0.91 & $(0.71,1.17)$ & 1.02 & $(0.79,1.33)$ \\
\hline & most deprived quartile & 0.60 & $(0.47,0.78)$ & 0.73 & $(0.55,0.96)$ \\
\hline Rurality (rural) & urban & 0.77 & $(0.63,0.95)$ & 0.88 & $(0.71,1.10)$ \\
\hline Area level availability (average $=50 \%$ ) & $10 \%$ increase & 0.98 & $(0.95,1.02)$ & 0.99 & $(0.96,1.03)$ \\
\hline \multicolumn{6}{|l|}{ Hospital characteristics } \\
\hline On site availability (none) & throughout study period & 5.71 & $(2.82,11.54)$ & 5.67 & $(2.63,12.20)$ \\
\hline
\end{tabular}

The average age of patients was 63 years and $54 \%(n=4221)$ were men. The majority $(66 \%)$ were married while $9 \%$ were single, $14 \%$ were widowed and $11 \%$ were classed as other, including divorced. In terms of area deprivation, the largest group of patients were resident in the most deprived areas in Scotland (28\%) and the smallest group were those resident in the most affluent (21\%). Just over $40 \%$ of patients were resident in rural areas. Almost $30 \%$ of patients were first admitted as an emergency and $84 \%$ of patients were first admitted to surgery. A total of 1568 (20\%) of the patients died within the six month treatment period.

DETERMINANTS OF CHEMOTHERAPY

Table 1 shows the effects of patient, area and hospital characteristics. The estimated odds ratio (OR) of receiving chemotherapy is reported both unadjusted and after adjustment for all other characteristics although, in general, there is little difference between the two sets of results. There was a strong effect of age with the treatment being more likely to be provided to younger patients. The odds of treatment were increased but not significantly for persons who were married compared with those who were single, although there was a larger increase in odds for the group including divorced patients. The odds of treatment were significantly decreased for persons who were widowed although this effect was attenuated by adjusting for other factors such as age. The difference in provision between men and women was not significant.

Patients who presented as an emergency were less likely to receive the treatment within six months than those admitted electively, although there was no apparent difference in provision for patients who died within six months of first admission and those who did not. In general, patients with comorbidities were less likely to receive chemotherapy than those with no comorbidities although the size of the effect varied according to the type of comorbidity. Only patients who had a cancer comorbidity were significantly more likely to receive chemotherapy than patients with no secondary diagnosis. The majority of this group $(76 \%)$ had a secondary malignant neoplasm (ICD9 196-198) implying a late stage presentation of the disease.

There was evidence of a threshold, area level deprivation effect. Patients living in the most deprived areas had significantly decreased odds of receiving chemotherapy compared with patients living in the most affluent areas while those living in mid-affluent and mid-deprived areas did not. Patients living in rural areas were more likely to receive the treatment than patients in urban areas although this difference was not significant once other characteristics were taken into account. Differential effects of deprivation were estimated for patients living in urban and rural areas but were not statistically significant.

The majority of patients resident in the same area were first admitted to the same hospital (median $=89 \%$ ), therefore, the two measures of availability at area and hospital level were highly correlated. For this reason, each of the adjusted effects of availability reported in table 1 was estimated in separate models. There was no significant association between area level availability and a patient's odds of receiving the treatment but there was a strong significant effect of on site availability at the hospital of first admission. Patients first admitted to a hospital providing on site chemotherapy throughout the study period were nearly six times more likely to receive the treatment themselves. Estimating the effects of each measure in the same model increased both the positive effect of on site provision at the hospital of first admission and the negative effect of area level availability, although this remained non-significant. This result highlighted the 


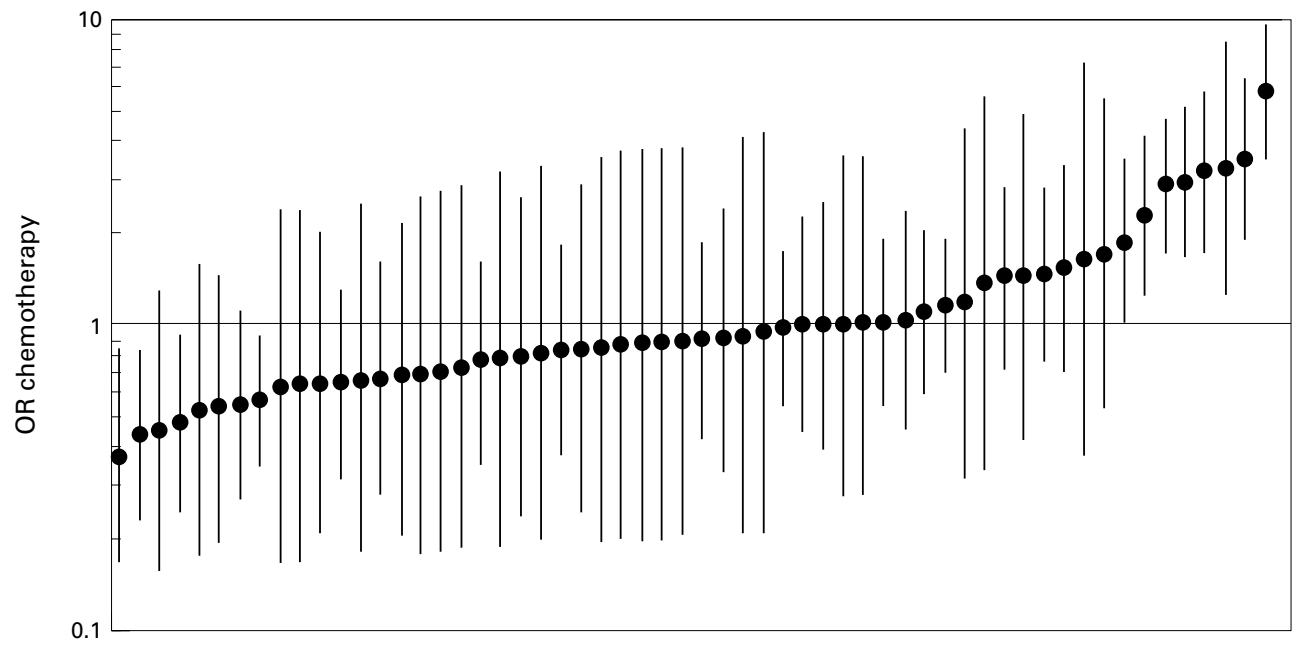

Figure 1 Additional unexplained effects on probability of chemotherapy associated with individual hospitals: point estimates and 95\% CI for 58 Scottish hospitals, Scottish average OR $=1$.

strong positive correlation between the measures and all subsequent results are based on models excluding area level availability.

AREA AND HOSPITAL VARIATION

Without adjusting for patient, area and hospital level characteristics there was significant variation between both area of residence and hospital of first admission, although the variation between hospitals was over six times that observed between areas. The variation between areas was entirely accounted for after adjusting for patient and area level characteristics. The variation between hospitals remained large even after adjustment for on site chemotherapy and decreased by $57 \%$ from the unadjusted to the completely adjusted model. However, a closer examination of the individual hospital effects showed a single hospital that appeared to be an outlier and that substantially increased the odds of chemotherapy for patients first admitted there $(\mathrm{OR}=11.2,95 \% \mathrm{CI} 4.6,27.1)$. It was reasonable to omit this hospital on the basis that relatively few patients were first admitted there $(n=22)$ and it had been closed by the end of the study period. The effects reported in table 1 were largely unaffected by its exclusion with the exception of the single hospital level variable. The hospital in question provided on site chemotherapy and the majority of patients first admitted there received the treatment $(68 \%)$, therefore, by excluding it, the effect of on site provision at the hospital of first admission decreased to $\mathrm{OR}=4.3,95 \%$ CI 2.2 , 8.6. Exclusion of this outlying hospital also had the effect of decreasing the between hospital variation by a further $27 \%$. Figure 1 shows the additional, unexplained hospital effects, which are expressed as ORs of receiving chemotherapy relative to the Scottish average $(\mathrm{OR}=1)$ and are shown with $95 \% \mathrm{CI}$. Of the remaining 58 hospitals, eight were found to significantly increase the odds of chemotherapy while the odds were significantly decreased for four. Each of these four hospitals, which in total first admitted $19 \%$ of the patient population, had on site availability for some or all of the five years of study. Around $23 \%$ of patients were first admitted to one of the eight hospitals increasing the odds of treatment but only two of them had on site availability.

UPTAKE OF CHEMOTHERAPY

It was useful to determine how variation was changing over time because the study period represents a time when chemotherapy was emerging as a new technology in the treatment of colorectal cancer. Uptake was investigated by incorporating a trend variable to estimate the annual average change in chemotherapy provision and by estimating how this trend varied between hospitals. The odds of chemotherapy increased annually with, for example, cases first treated in 1993 being 50\% more likely to receive chemotherapy than cases first treated in 1992. There was also evidence that between hospital variation was decreasing over time as a result of the rate of uptake being greater in hospitals for which the rate of chemotherapy treatment was low in comparison with the Scottish average. This resulted in the unexplained variation between hospitals of first admission decreasing by $22 \%$ between 1990 and 1994.

\section{Discussion}

This paper has illustrated how to identify and monitor variations in access to chemotherapy for colorectal cancer in Scotland. It has demonstrated that characteristics of the patients, the areas in which they are resident, and the hospitals to which they are referred impact upon the type of treatment they receive. Further to this it has shown that the hospital to which a patient is first admitted can influence the type of treatment received regardless of whether there is on site provision of that treatment. The variation observed between hospitals decreased over the study period but remained significant in 1994.

The utility of routine data for monitoring variations in access to health care is largely dependent on the quality of those data. In general, the number of cases identified from the linked SMR1 data set corresponded well with cancer registration statistics for the same 
Table 2 Distribution of severity indicators by deprivation quartile

\begin{tabular}{|c|c|c|c|c|}
\hline & $\begin{array}{l}\text { Most affluent } \\
\text { quartile \% }\end{array}$ & $\begin{array}{l}\text { Mid-affluent } \\
\text { quartile \% }\end{array}$ & $\begin{array}{l}\text { Mid-deprived } \\
\text { quartile \% }\end{array}$ & $\begin{array}{l}\text { Most deprived } \\
\text { quartile\% }\end{array}$ \\
\hline \multicolumn{5}{|l|}{ Secondary diagnosis } \\
\hline other neoplasms & 20 & 17 & 19 & 15 \\
\hline other & 15 & 17 & 16 & 16 \\
\hline \multicolumn{5}{|l|}{ Type of admission } \\
\hline emergency & 27 & 32 & 31 & 30 \\
\hline \multicolumn{5}{|l|}{ Died within 6 months } \\
\hline no & 84 & 81 & 80 & 77 \\
\hline yes & 16 & 19 & 20 & 23 \\
\hline
\end{tabular}

period and the fact that most of the increase in hospital activity could be attributed to chemotherapy episodes suggests that the data were capturing the changing patterns of care. It is unlikely that differences according to patient and area characteristics could be attributed to coding errors although variations are known to exist between hospitals in the recording accuracy of diagnoses and procedures. ${ }^{18}$ It is possible that some of the hospital effects could be attributable to more stringent coding practices, especially as chemotherapy will not always be the principal procedure, or to different organisation of services such as the use of outpatients for the provision of the treatment. In practice, feedback to hospitals should help to establish whether an apparent underprovision or over-provision of services is genuine or misrepresented by the available data. In this example, because of the linked data, chemotherapy was measured at the patient level so reducing the impact of hospital measurement error. This is supported by the fact that the majority of hospitals associated with an increase in the odds of chemotherapy did not in fact provide (that is, record) the treatment themselves.

The impact of availability or provision was explored using two measures derived from the routine data and it is important to examine the ability of these measures to explain variations between hospitals and areas. Unlike the findings reported by Gatsonis et al, there was no significant effect of area level availability. This result may be explained by the fact that our measure of availability was based on small numbers of events and was less robust as a measure of locally available services compared with a study based on larger areas. While on site availability at the hospital of first admission was found to impact upon the likelihood of receiving chemotherapy, adjusting for this explained relatively little of the between hospital variation. It is likely that as chemotherapy becomes more widely recognised as a treatment for colorectal cancer, not only will the impact of on site provision decrease but the number of hospitals that provide chemotherapy will increase.

The lack of information on stage of disease at presentation made it difficult to adjust for clinical need for the service. There is evidence that socioeconomic deprivation is associated with later presentation of the disease and one recent Scottish study found that patients living in the most deprived areas were more likely than others to present with local advanced disease. ${ }^{5}$ Using information on secondary diagnoses as a proxy for disease staging, our data did not support this (table 2); patients living in the most deprived areas of Scotland were least likely to have a recorded cancer comorbidity. These results highlight the inadequacy of recorded secondary diagnoses as measures of disease severity and, unless there is systematic bias in the recording of comorbidities, do not explain the decreased adjusted odds of chemotherapy associated with deprivation. Table 2 does show that mortality increases with deprivation and, while there is no clear gradient, there are fewer emergency admissions for patients resident in the most affluent areas; these indicators may support the finding of later presentation for more deprived patients. It is plausible that the results found in terms of area deprivation may reflect genuine inequity of access to chemotherapy for colorectal cancer with, for example, patients resident in these areas having inadequate means of transport to attend repeat follow up visits.

The finding of decreased utilisation for older patients may reflect clinical judgement regarding the expected gain in quantity or quality of life balanced with the toxicity of the treatment. The trend with age and the lower rate of treatment in single persons may, however, reflect patient choice as recent studies show that it is younger patients and those with families who are more willing to accept chemotherapy for a limited clinical benefit. ${ }^{19}$ Indeed, it is important to underline that the results reported in this paper represent a time when the clinical benefits of chemotherapy, although promising, were not yet fully established. The strong effects of hospital of first admission are likely to be proxy measures of consultant effects that reflect their own knowledge, experience and belief in the benefits proffered by chemotherapy, and it is to be expected that these effects should decrease now that guidelines have been issued.

Many thanks to A H Leyland and F A Boddy for their advice throughout and to the anonymous referees for their helpful comments.

Funding: this research was supported by the Chief Scientist Office at the Scottish Office Department of Health. Opinions Office at the Scottish Office Department of Health. Opinions
and conclusions expressed in this paper are not necessarily those of the Scottish Office Department of Health.

of the Scottish Office Depa
Conflicts of interest: none.

1 Seventh King's Fund Forum. Cancer of the colon and rectum consensus statement. London: King's Fund, 1990.

2 Moertel CG, Fleming TR, MacDonald JS, et al. Levamisole and fluorouracil for adjuvant therapy of resected colon carand fluorouracil for adjuvant therapy of
cinoma. N Engl f Med 1990;322:352-8.

3 Scottish Intercollegiate Guidelines Network. Colorectal cancer. A national clinical guideline recommended for use in Scotland. Edinburgh: SIGN, 1997.

4 NHS Centre for Reviews and Dissemination. The management of colorectal cancer. Effective Health Care 1997;3:1-12.

5 Ionescu MV, Carey F, Tait IS, et al. Socioeconomic status and stage at presentation of colorectal cancer. Lancet 1998; 352:1439.

6 Mandelblatt J, Andrews H, Kao R, et al. The late stage diagnosis of colorectal cancer: demographic and socioeconomic factors. Am f Public Health 1996;86:1794-7.

7 Harris V, Sandridge AL, Black RJ, et al. Cancer Registration Statistics Scotland 1986-1995. Edinburgh: ISD Scotland Publications, 1998.

8 Pollock AM, Vickers N. Trends in colorectal cancer care in southern England, 1989-1993: using HES data to inform cancer services reviews. $\mathcal{F}$ Epidemiol Community Health 1998;52:433-8.

9 Benzeval M, Judge K, Whitehead M. The role of the NHS. In: Benzeval M, Judge K, Whitehead M, eds. Tackling inequalities in health: an agenda for action. London: King's Fund, 1995. 
10 Goddard M, Smith P. Equity of access to health care. York: University of York, Centre for Health Economics, 1998.

11 Ben-Shlomo Y, Chaturvedi N. Assessing equity in access to healthcare provision in the UK: does where you live affect your chances of getting a coronary artery bypass graft? $\mathcal{F}$ Epidemiol Community Health 1995;49:200-4.

12 Gatsonis CA, Epstein AM, Newhouse JP, et al. Variations in the utilization of coronary angiography for elderly patient with an acute myocardial infarction. Med Care 1995;33: 625-42.

13 Kendrick S, Clark J. The Scottish Record Linkage System. Health Bull (Edinb) 1993;51:72-9.

14 Office of Population Censuses and Surveys. Classification of surgical operations and procedures, 4th revision. London: OPCS, 1987.
15 Carstairs V, Morris R. Deprivation and health in Scotland. Aberdeen: Aberdeen University Press, 1991.

6 Rice N, Leyland AH. Multilevel models: applications to health data. Fournal of Health Services Research and Policy 1996;1:154-164.

17 Rasbash J, Goldstein H. Efficient analysis of mixed hierarchical and cross-classified random structures using a multilevel model. Fournal of Educational Statistics 1994;19: 337-50.

18 Harley K, Jones C. Quality of Scottish Morbidity Record (SMR) data. Health Bull (Edinb) 1996;54:410-17.

19 Redmond K. Assessing patient's needs and preferences in the management of advanced colorectal cancer. $\mathrm{Br} F$ Cancer 1998;77 (suppl 2):5-7. 